\title{
Marker enrichment and construction of haplotype-specific BAC contigs for the polyembryony genomic region in Citrus
}

\author{
Michiharu Nakano*1), Tokurou Shimizu ${ }^{2}$, Hiroshi Fujiii), Takehiko Shimada'), Tomoko Endo²), \\ Hirohisa Nesumi ${ }^{2)}$, Takeshi Kuniga ${ }^{2,3)}$ and Mitsuo Omura ${ }^{4)}$ \\ 1) The United Graduate School of Agricultural Science, Gifu University, 1-1 Yanagido, Gifu 501-1193, Japan \\ 2) Department of Citrus Research, National Institute of Fruit Tree Science, 485-6 Okitsu-nakacho Shimizu, Shizuoka 424-0292, Japan \\ 3) Present address: National Agricultural Research Center for Western Region Shikoku Research Center, 2575 Ikano, Zentsuuji, Kagawa \\ 765-0053, Japan \\ 4) Faculty of Agriculture, Shizuoka University, 836 Ohya, Suruga, Shizuoka 422-8529, Japan
}

Polyembryony, in which zygotic and apomictic embryos develop concurrently in a seed, is widespread in Citrus. To obtain further genomic information about the previously characterized map position of the polyembryony locus, the relationship among linkage maps from 3 different hybrid populations was determined using common markers flanking the polyembryony locus. By mapping the polyembryony locus with cleaved amplified polymorphic sequence (CAPS) markers in a F180 × Harumi' population, the polyembryony locus was detected in a high-density marker region co-segregated to marker Mf0086. By screening a bacterial artificial chromosome (BAC) library with marker Mf0086, two contigs reflecting the polyembryony and monoembryony haplotype were constructed. The single nucleotide polymorphism (SNP) markers detected on these contigs were mapped on the nearest position to the polyembryony locus in linkage group 1 of 'Miyagawa wase' and 'Harumi'. The highly conserved marker lineage in the flanking regions of the polyembryony locus in these polyembryonic cultivars of different parentage suggested that the genomic region of the polyembryony locus might have a common structure in a wide range of Citrus cultivars.

Key Words: Citrus, polyembryony, genetic mapping, CAPS, SNP.

\section{Introduction}

Apomixis, the process of reproduction by means of asexually produced seeds, is a common reproductive system in plants, occurring in $\sim 400$ genera from $\sim 40$ families (Carman 1997). In apomictic plant species, somatically developed embryos derived from maternal tissues are formed in a seed. Two major types, gametophytic and sporophytic, are recognized according to the somatic embryo developmental process (Koltunow 1993). Polyembryony is a sporophytic type of apomixis in which somatic embryos derived from nucellar tissues of ovules form concurrently with zygotic embryos in a seed (Kobayashi et al. 1978, 1979). Polyembryony is a common trait in the genus Citrus. Ueno et al. (1967) showed that it occurred in 85 of 116 cultivars of Citrus and related genera examined. Regarding the genetic basis of polyembryony, several authors concluded that a single, or a few, dominant genes were involved in the determination of embryo type from the observation of segregation in various crosses between monoembryonic and polyembryonic cultivars, as well as crosses among polyembryonic cultivars (Parlevliet

Communicated by T. Yamamoto

Received May 29, 2008. Accepted September 8, 2008.

*Corresponding author (e-mail: minakano@affrc.go.jp) and Cameron 1959, Iwamasa et al. 1967, Hong et al. 2001); however, the genes regulating polyembryonic embryogenesis in Citrus have not been identified yet.

The recent development of DNA markers has been applied, for example, to construct genetic maps, to obtain selection markers for breeding, and to genotype genetic resources. In such analyses, various DNA markers, for example, random amplified polymorphic DNA (RAPD), cleaved amplified polymorphic sequence (CAPS) and single nucleotide polymorphism (SNP) markers, have been used. DNA markers linked to important traits have been developed for marker-assisted selection (MAS) and markerassisted typing of cultivars in Citrus (Gmitter et al. 2007). Genetic linkage maps in Citrus and related genera have been previously constructed by several research groups (Durham et al. 1992, Jarrell et al. 1992, Omura et al. 2003, Bernet et al. 2004, Carlos de Oliveira et al. 2007, Chen et al. 2008). Application of such genetic maps could also allow identification of the genes regulating agriculturally important traits through map-based cloning.

In a previous study, mapping of a polyembryony locus was performed on a segregating population derived from a cross between the Citrus cultivars 'Kiyomi' (Citrus unshiu Marcow. 'Miyagawa wase' $\times C$. sinensis (L.) Osbeck 'Trovita') $\times$ 'Miyagawa wase') $\times$ 'Miyagawa wase' (Nakano 
et al. 2008). By bulked segregation analysis with RAPD markers and mapping on the previously constructed linkage map of the population (Omura et al. 2003), the polyembryony locus was detected on linkage group K-9, which consisted of five RAPD markers and two expressed sequence tag (EST)-derived CAPS markers (Nakano et al. 2008). The result supported the hypothesis of monogenic control of polyembryony in the 'Kiyomi' $\times$ 'Miyagawa wase' population; however, the marker density of the linkage group is still low and additional markers on the map are required to permit further analysis.

A bacterial artificial chromosome (BAC) library with a long genomic DNA insert (Shizuya et al. 1992) has been used to obtain genomic information for the candidate region determined by genetic mapping. In studies of horticultural crops, the BAC library system was utilized to construct physical maps for the $V f$ scab-resistant gene in apple (Patocchi et al. 1999), $R f_{l}$ fertility-restoring gene in cotton (Yin et al. 2006), and sex-determining $M$-locus in asparagus (TelgmannRuber et al. 2007). BAC libraries from Poncirus trifoliata (L.) Raf., a relative of Citrus (Yang et al. 2001), and the Citrus $\times$ Poncirus hybrid USDA 17-47 (Deng et al. 2001) were used to construct physical maps covering the gene conferring resistance to Citrus tristeza virus (Yang et al. 2001, 2003). In addition, a BAC library from 'Miyagawa wase' (Shimada et al. 2005) was also constructed. Determination of the sequence of the genomic region in BAC clones could also contribute to refinement of the genetic map structure for the polyembryony locus in Citrus.

In the present study, we mapped the polyembryony locus in the segregating population F180 ('Kiyomi' $\times$ 'Wilking' ('King' $\times$ Mediterranean mandarin $(C$. deliciosa Ten.))) $\times$ 'Harumi' ('Kiyomi' $\times$ C. reticulata Blanco Ponkan F-2432) using EST-based CAPS markers as well as newly developed SNP markers derived from BAC end sequences (BES). By comparing multiple genetic linkage maps with common DNA markers, the common map structure flanking the polyembryony locus was confirmed. The genomic structure obtained in the present study will contribute to future investigations of the genes determining polyembryony through map-based cloning and to our understanding of the molecular structure of the polyembryony locus.

\section{Materials and Methods}

\section{Plant material}

Three segregating populations were utilized in this study (Fig. 1). The first population (KM) consisted of 41 individuals from a cross between 'Kiyomi' and 'Miyagawa wase', which was also utilized in a previous study (Nakano et al. 2008). The second population (AG) consisted of 87 hybrid individuals derived from a cross between 'Okitsu 46 Gou' (A255: 'Sweet Spring' (C. unshiu 'Ueda unshiu' $\times$ C. hassaku hort. ex Tanaka) $\times$ 'Trovita') and 'Kankitsu Chukanbohon Nou 5 Gou' (G434: 'Lee' (C. clementina hort. ex Tanaka × 'Orlando' (C. paradisi Macf. 'Duncan’ $\times$

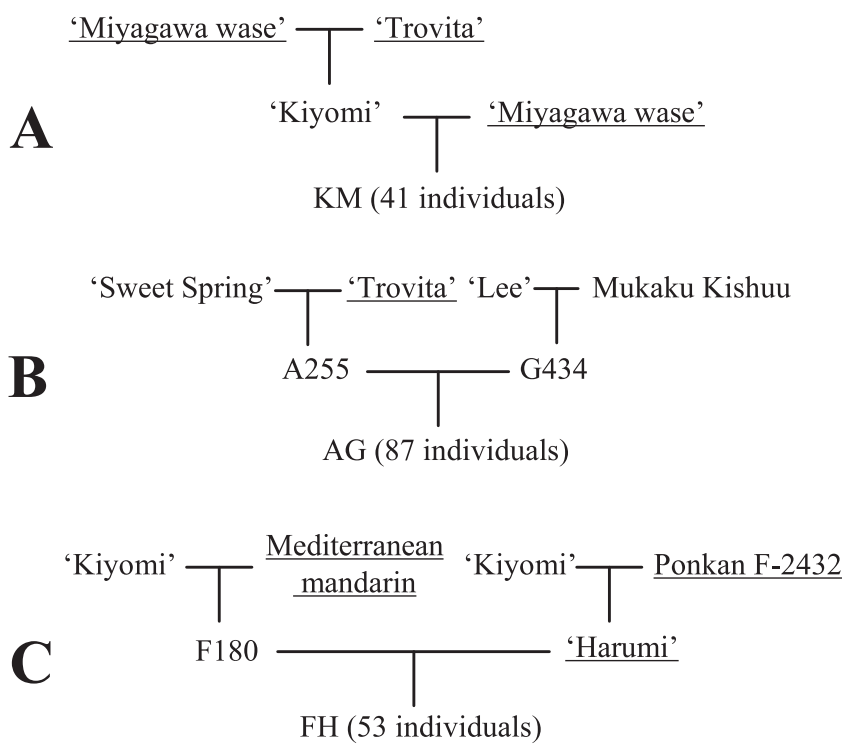

Fig. 1. Segregating populations used in this study. A: 'Kiyomi' $\times$ 'Miyagawa wase', B: A255 × G434, C: F180× 'Harumi'. Polyembryonic cultivars are underlined.

C. tangerina hort. ex Tanaka) $) \times C$. kinokuni hort. ex Tanaka 'Mukaku Kishuu'). This population was developed to construct a high-density linkage map using EST-based CAPS markers (Omura et al. in preparation). The third population (FH), consisting of 53 individuals from a cross between F180 and 'Harumi', was developed to evaluate the segregation of polyembryony in the present study. The FH population was raised in 1996 and top-grafted on interstock to reduce the time to fruiting. All of the segregating populations and parental cultivars were raised and grown at the National Institute of Fruit Tree Science, Department of Citrus Research, Okitsu, Shimizu-ku, Shizuoka, Japan. Total genomic DNA was isolated from each individual and parental cultivar for all segregating populations according to the CTAB-based method (Shimada et al. 2000) and subjected to DNA marker analysis.

\section{Evaluation of polyembryony segregation}

Fruits derived from open pollination of individuals in the FH population were harvested and seeds were collected from five fruits per individual in November 2005. The embryo number in each seed was counted with the aid of a stereoscopic microscope. The embryo type for each seed was classified as either monoembryonic or polyembryonic. The embryo type was investigated in 10 seeds for individuals producing only polyembryonic seeds and in 20 seeds for individuals producing only monoembryonic seeds. The embryo type for each individual is represented numerically by the ratio of the number of polyembryonic seeds per total seed number observed.

\section{Marker analysis}

The RAPD analysis followed the methodology of 
Nakano et al. (2008). In the CAPS analysis, PCR amplification followed by restriction enzyme digestion was performed to detect polymorphisms. PCR amplification was performed in a total volume of $10 \mu \mathrm{l}$ containing $10 \mathrm{ng}$ of genomic DNA, $0.3 \mu \mathrm{M}$ of each primer (Table 1 ), $0.16 \mathrm{mM}$ of each $\mathrm{dNTP}$, 0.25 unit AmpliTaq Gold DNA polymerase, $2.5 \mathrm{mM} \mathrm{MgCl}_{2}$, and $1 \times$ PCR buffer II (Applied Biosystems, Foster City, CA, USA). After denaturation at $94.5^{\circ} \mathrm{C}$ for $10 \mathrm{~min}$, the PCR reaction consisted of 35 cycles of $94^{\circ} \mathrm{C}$ for $40 \mathrm{sec}$, annealing for $1 \mathrm{~min}$ (see Table 1 for temperature), $72^{\circ} \mathrm{C}$ for $2 \mathrm{~min}$, and with the final extension at $72^{\circ} \mathrm{C}$ for $7 \mathrm{~min}$. The $\mathrm{PCR}$ products were subjected to restriction enzyme digestion using $3 \mu \mathrm{l}$ PCR product, $1 \times$ corresponding buffer, and 0.8-2.0 units of restriction enzyme in a volume of $10 \mu \mathrm{l}$. The products were electrophoresed on an agarose gel buffered with $1 \times$ TAE at $100 \mathrm{~V}$ and visualized by EtBr staining. To obtain cultivarspecific CAPS markers, the following restriction enzymes were tested: Msp I, Hae III, Hinc II, Rsa I, Pvu II, Sty I, Hinf I, Mlu I, EcoR I, EcoR V, Hha I, Mbo I, Dra I, Xba I, Hind III, and BamH I (Nippon Gene, Tokyo, Japan). CAPS markers differing between parental cultivars were selected for segregation analysis. The restriction enzymes used to detect the polymorphism for each marker are listed in Table 1. Data on the mapping of CAPS markers in the AG population will be included in a forthcoming publication.

In the SNP analysis, PCR products for each individual were purified using UNIFILTER GF/F (Whatman, Kent, UK), directly sequenced with specific primers using the BigDye Terminator v. 3.1 cycle sequencing kit and resolved on an ABI PRISM 3100 Genetic Analyzer (Applied Biosystems). Sequence analysis was performed with Pregap4, Gap4, and Trev contained in the Staden package 1.6.0 (http://sourceforge.net/projects/staden). Sequences were processed using pregap4 with 'Quality clip' $(\mathrm{QV}>15)$ and the filtered sequences were assembled using Gap4 with $20 \%$ maximum mismatch. SNP sites visually confirmed with Trev were scored on a spreadsheet. For each marker-specific and BES-specific primer, the genomic PCR products of 'Miyagawa wase' were sequenced and heterozygous SNP sites on each amplification product were scored. Of these SNP sites, those heterozygous for 'Miyagawa wase' and homozygous for 'Kiyomi' were subjected to linkage analysis in the KM population, and SNP sites heterozygous for 'Harumi' and homozygous for F180 were subjected to linkage analysis in the $\mathrm{FH}$ population.

\section{Linkage analysis}

Linkage analysis was performed with JoinMap 3.0 (Plant Research International, Wageningen, The Netherlands). Mapping analysis was conducted using a minimum LOD score of 4.0 in cross-pollination mode in all populations. In the AG population, the segregation of RAPD markers R1011 and R1026, which were mapped on linkage group K9 in a previous study (Nakano et al. 2008), were analyzed with marker data for the AG population. In the FH population, CAPS and SNP markers heterozygous for 'Harumi' and homozygous for F180 were selected to construct a linkage map for 'Harumi'. The segregation of markers in 47 individuals with either a $0 \%$ or $100 \%$ polyembryonic seed ratio was analyzed. In the KM population, newly developed SNP markers for Lp0211, Ks9001, 006B09M, and 048F02T were analyzed in conjunction with previous data on the linkage group K-9 (Nakano et al. 2008).

\section{Screening and end-sequencing of BAC clones}

A BAC library consisting of 36,864 clones $(384 \times 96$-well plates) for C. unshiu 'Miyagawa wase' was previously constructed with the pECBAC1 vector, and the genome coverage was estimated as 13.3 times that of the haploid genome (Shimada et al. 2005). The pooled library was screened with CAPS marker Mf0086-specific primers by PCR under the same conditions used for CAPS analysis. From PCRpositive BAC clones, plasmid DNA was extracted using the standard alkaline SDS method, and $100 \mathrm{ng}$ DNA was used for sequencing with both the T7 promoter and M13 reverse primers. The sequences were trimmed by pregap4 with 'Quality clip' (QV>15) and 'Sequencing vector clip', and subjected to BLAST searches of DDBJ ALL with BLASTN

Table 1. CAPS and BES primer sequences, annealing temperatures and the restriction enzyme used to detect polymorphism for each marker

\begin{tabular}{|c|c|c|c|c|}
\hline Marker & Forward sequence $\left(5^{\prime}-3^{\prime}\right)$ & Reverse sequence $\left(5^{\prime}-3^{\prime}\right)$ & Annealing temp $\left({ }^{\circ} \mathrm{C}\right)$ & Restriction enzyme \\
\hline A10636 & AAAGATTGGCCACTATTTTGA & GGGCGATTGCTTATTTTGT & 56 & EcoR I \\
\hline $\mathrm{Fb} 0103$ & CAAGTGCCCCCATTGA & TACTGCCACACTCCATAACAC & 58 & Msp I \\
\hline Gn0011 & CTGAAGTCTTTCCATCCCGA & GAGAGCTTCCTCAATGTCCA & 54 & Hinc II \\
\hline Gn0017 & GCAGTGATCTCTACTATTCAACTC & TTTGTGTAATAGTCTCATGCGG & 60 & Hae III \\
\hline Ks9001 & AGCCTTCTTGCGTCAGTA & TCGTTAAAGTTACCCAATGTT & 60 & Hae III \\
\hline Lp0211 & CCGAAGCACAGGAAACC & ACATACAATGCGACAAATCAT & 54 & EcoR I \\
\hline Lp0237 & TGTGCTGCCCTTGTGGTTG & GCAGCCCCGACTCTTTCATTA & 58 & $P v u$ II \\
\hline Mf0086 & TCAACAACAGCTAATACTCGC & TACCAGTAGACAGCCGTCAGC & 56 & Dra I \\
\hline TF0049 & TCCACCAACAGCCAAGTACGA & TTCTTTGCGGAAGTAGTAGAC & 62 & Hae III \\
\hline Wy0028 & GGAGGTGCCGAAGAAGAAAA & GCTTAGCTATGGCCGATGTATGA & 58 & Dra I \\
\hline 006B09M & TCATTACCTATTGCTTAGGGTG & GTAATGACAATTGGTGCTTACG & 60 & \\
\hline 006B09T & TTTGGCTTCTTCATTACCTG & GTTACTGAATGCTGGTCCTC & 60 & \\
\hline 048F02M & ACGTTATCCATGGGTTTGATACTC & ATGGGTAAGAGGAAATTGATGG & 60 & \\
\hline 048F02T & CAACAAGACCCAAGTAATAAGCAC & CTTCGTTTAGTAGAGCATCACC & 60 & \\
\hline
\end{tabular}


and of the protein default data (UniProt + PRF + PDB) with BLASTX (http://blast.ddbj.nig.ac.jp/top-j.html, November 1, 2006). Sequences that did not show homology to transposable elements were used to design specific primer pairs with PerlPrimer (http://perlprimer.sourceforge.net/) with the criterion $\mathrm{Tm}=58-62^{\circ} \mathrm{C}$.

\section{$B A C$ contig construction}

The BAC library was further screened with two specific primer sets $(006 \mathrm{~B} 09 \mathrm{M} / \mathrm{T}, 048 \mathrm{~F} 02 \mathrm{M} / \mathrm{T}$; M: M13-reverse end-specific, T: T7 end-specific) designed from the end sequences of two BAC clones 006B09 and 048F02. For each primer set, 6 to $10 \mathrm{BAC}$ clones were isolated. Of the $\mathrm{BAC}$ clones screened, 8 clones were selected and their overlapping patterns were determined by fingerprinting and PCR amplification with BES primers. Amplification products were directly sequenced and SNP markers were scored for heterozygous SNP sites on genomic PCR products of 'Miyagawa wase'. Based on the sequence of each SNP site, BAC clones were classified into two groups. For fingerprinting, 100 ng Hind III-digested BAC DNAs were electrophoresed on an agarose gel buffered with $1 \times \mathrm{TAE}$ at $70 \mathrm{~V}$ for 8 hours and stained with EtBr. The gel was scanned with a FMBIOII (Hitachi Software Engineering, Tokyo, Japan), and fragment sizes were estimated by ImageAnalysis (Hitachi Software Engineering). Overlaps among BAC clones were manually detected by matching restriction fragments.

\section{Results}

\section{Segregation of polyembryony in the FH population}

Segregation of polyembryony was analyzed in the $\mathrm{FH}$ population, which was derived from a cross between monoembryonic F180 and polyembryonic 'Harumi'. The embryo number of 53 individuals in the population was investigated in 2005. The polyembryonic seed ratio for each individual of the FH population was classified as shown in Figure 2. The monoembryonic seed parent F180 was scored as $0 \%$ polyembryonic and the polyembryonic pollen parent 'Harumi' was scored as $100 \%$. Among the hybrid population, 24 individuals were $0 \%, 23$ individuals were $100 \%$, and the remaining 6 individuals had intermediate scores (Fig. 2). The segregation of individuals classified as $0 \%$ or $100 \%$ fitted a $1: 1$ ratio, suggesting the segregation of a major gene determining the polyembryony $(100 \%)$ or monoembryony $(0 \%)$ phenotypes and of additional genes with minor effects on the polyembryonic seed ratio. In the present study, to test for a major gene effect on polyembryony, individuals classified as either $0 \%$ or $100 \%$ polyembryonic seed ratio were utilized for mapping.

Mapping of the polyembryony locus with the markers on linkage group 1

In a previous study, the polyembryony locus was mapped on a linkage group (K-9) in the segregating population 'Kiyomi' × 'Miyagawa wase' with five RAPD and two EST-

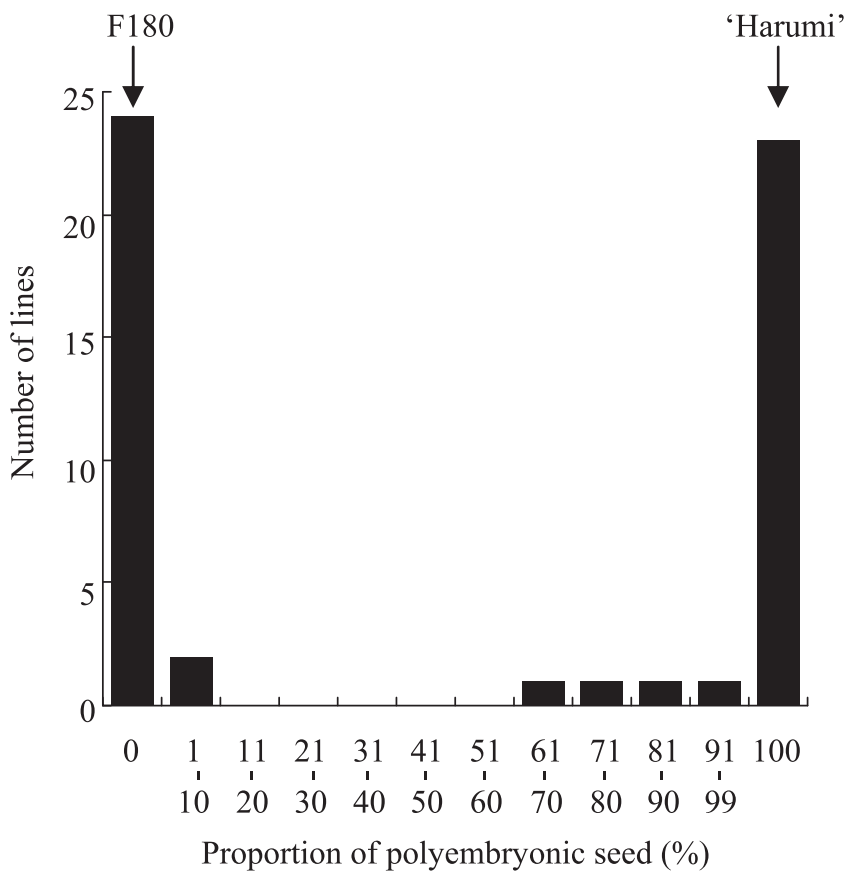

Fig. 2. Frequency distribution of the proportion of polyembryonic seeds in the FH population. The classes to which the parental cultivars F180 and 'Harumi' belong are indicated with an arrow.

based CAPS markers (Nakano et al. 2008). To obtain further markers flanking the polyembryony locus, markers mapped on K-9 were applied to the AG population, for which a highdensity genetic linkage map consisting of a large number of EST-based CAPS markers has been constructed (Omura et al. in preparation). Of the markers mapped on K-9, two RAPD markers (R1011 and R1026) and two EST-based CAPS markers (Gn0032 and Fb0103) were polymorphic in G434 (one of the parents of the AG population). By linkage analysis of these markers with marker data for the AG population, these markers were mapped on linkage group G-1 (Fig. 3, markers underlined). Their positions were as follows: R1011 was located at $0 \mathrm{cM}, \mathrm{R} 1026$ at $17.1 \mathrm{cM}$, Gn0032 at $22.5 \mathrm{cM}$, and $\mathrm{Fb} 0103$ at $27.3 \mathrm{cM}$ (Fig. 3). The map position of markers R1026 and Gn0032 was reversed on $\mathrm{G}-1$, but the sharing of markers on the overall linkage group suggests that linkage group K-9 corresponds to linkage group G-1 of G434. Several markers on G-1 were also mapped on linkage group A-1 of A255, the other parent of the AG population, and on linkage group K-1 of 'Kiyomi', one parent of the KM population. This also indicated that $\mathrm{K}$ 9 corresponds to linkage group 1 of 'Miyagawa wase'. Thus, linkage group K-9 was renamed M-1 in this study. In the AG population, segregation of polyembryony was not analyzed, but the linkage relationship of the markers suggests that the polyembryony locus might be linked to common markers on linkage group A-1/G-1; therefore, the markers on linkage group 1 in both $\mathrm{AG}$ and $\mathrm{KM}$ populations were selected and linkage with the polyembryony locus in the FH population was analyzed. 


\section{G-1}
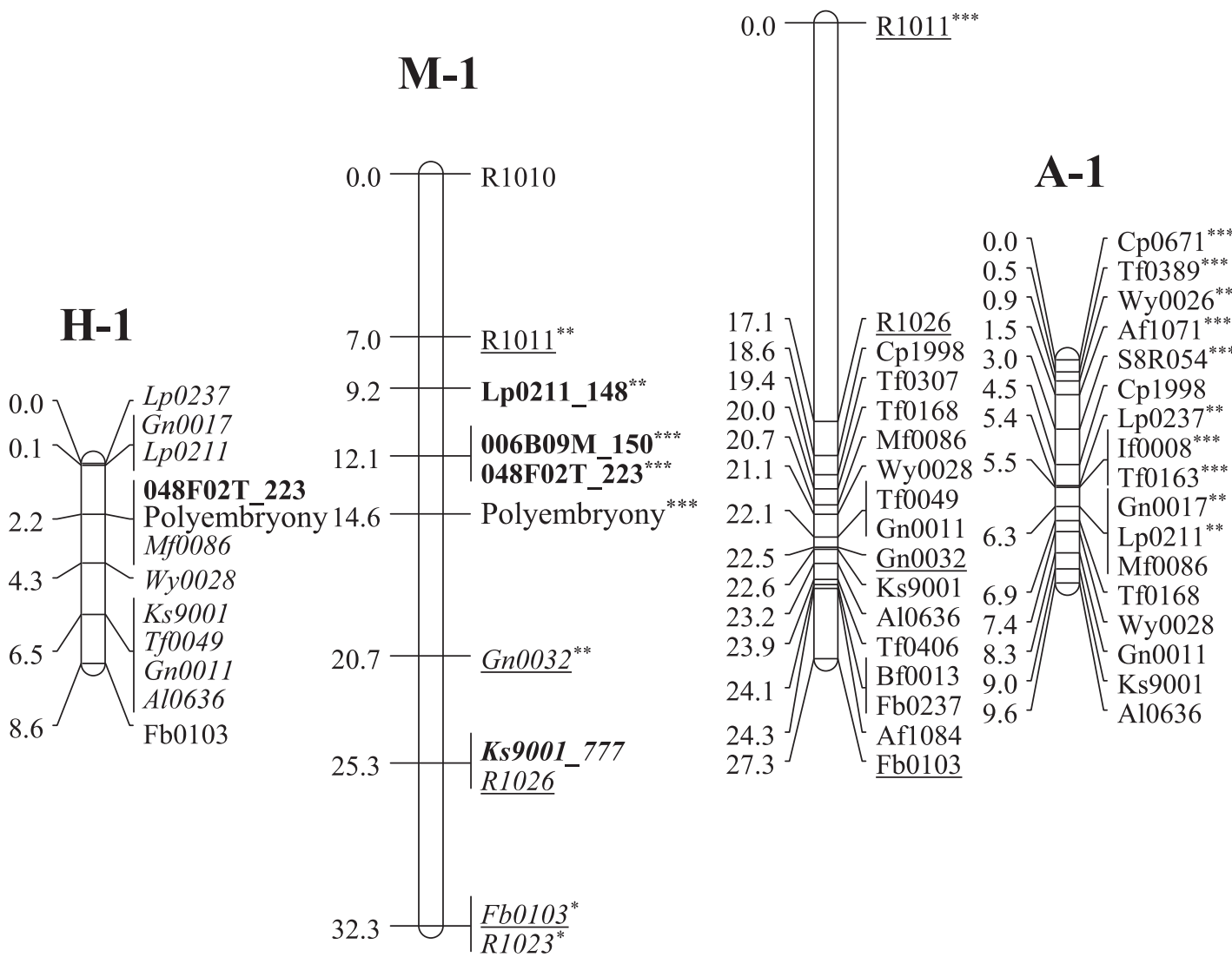

\section{K-1}

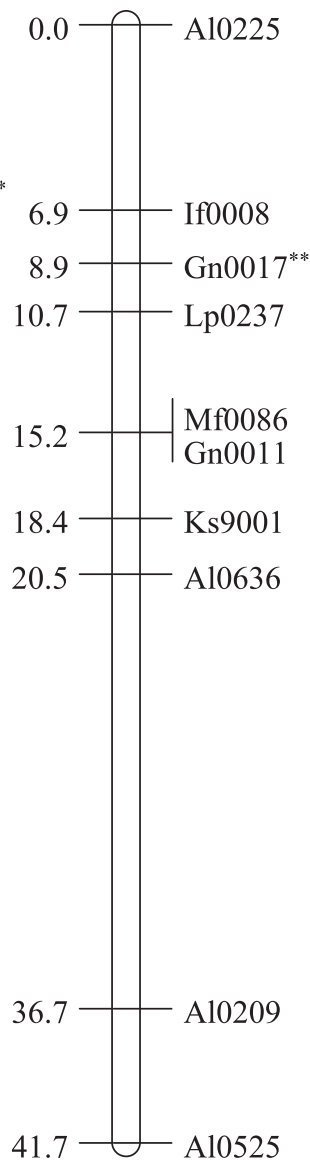

Fig. 3. Comparative map of linkage group 1 among different segregating populations. Distances between markers in centimorgan (Kosambi) are shown to the left of each linkage group. Linkage groups are designated as A: A255, G: G434, H: 'Harumi', K: 'Kiyomi', M: 'Miyagawa wase'. Linkage group M-1 of 'Miyagawa wase' corresponds to K-9 in the previous study of Nakano et al. (2008). Markers mapped on linkage groups M1 and G-1 are underlined. SNP markers are highlighted in bold. Marker type and designation are as follows: R: RAPD marker, Marker name_position: SNP markers, others: EST-derived CAPS markers. Asterisks indicate markers showing distorted segregation in a chi-square test. Distortions at $5 \%, 1 \%$ and $0.1 \%$ levels of significance are indicated as $* * *$, and $* * *$, respectively. Markers linked in repulsion phase to the polyembryony locus are italicized.

In the FH population, segregation of the polyembryony allele derived from 'Harumi' is expected (Fig. 1). To analyze the linkage of CAPS markers with the polyembryony locus in the FH population, markers that were heterozygous for 'Harumi' and homozygous for F180 were selected. Of the markers on linkage group 1, 10 markers (Lp0237, Lp0211, Gn0017, Mf0086, Wy0028, TF0049, Ks9001, Gn0011, Al0636, and Fb0103) showed polymorphism heterozygous for 'Harumi' and were applied to genotype 47 individuals of the $\mathrm{FH}$ population that were classified as either $0 \%$ or $100 \%$ polyembryonic seed ratio. From these results, the polyembryony locus was mapped in the $4.2 \mathrm{cM}$ interval between Lp0211 and Wy0028, and was completely linked with marker Mf0086 (Fig. 3). Of the markers on the linkage group, all except $\mathrm{Fb} 0103$ were linked in the repulsion phase to the polyembryony locus.

\section{BAC contig construction for the Mf0086 marker region}

To obtain additional genomic information on the regions flanking the polyembryony locus, the 'Miyagawa wase'derived BAC library was screened with marker Mf0086. After isolation of Mf0086 containing BAC clones, further screening was performed with BES-derived specific primers $(006 \mathrm{~B} 09 \mathrm{M} / \mathrm{T}$ and $048 \mathrm{~F} 02 \mathrm{M} / \mathrm{T})$, based on both end sequences of BAC clones 006B09 and 048F02. The alignment of BAC clones by the PCR amplification pattern of BES-specific primers is shown in Fig. 4A.

In the amplification products for 'Miyagawa wase' 
A

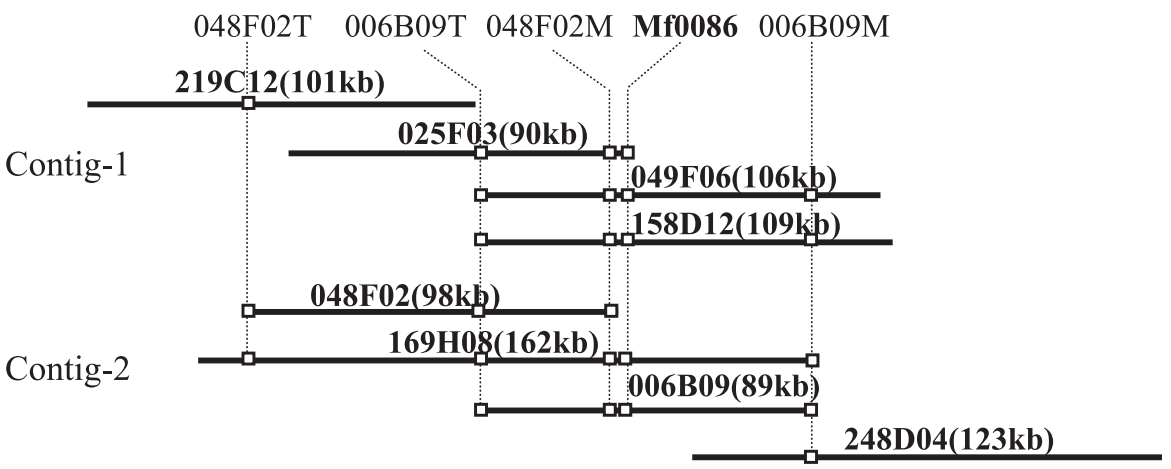

B

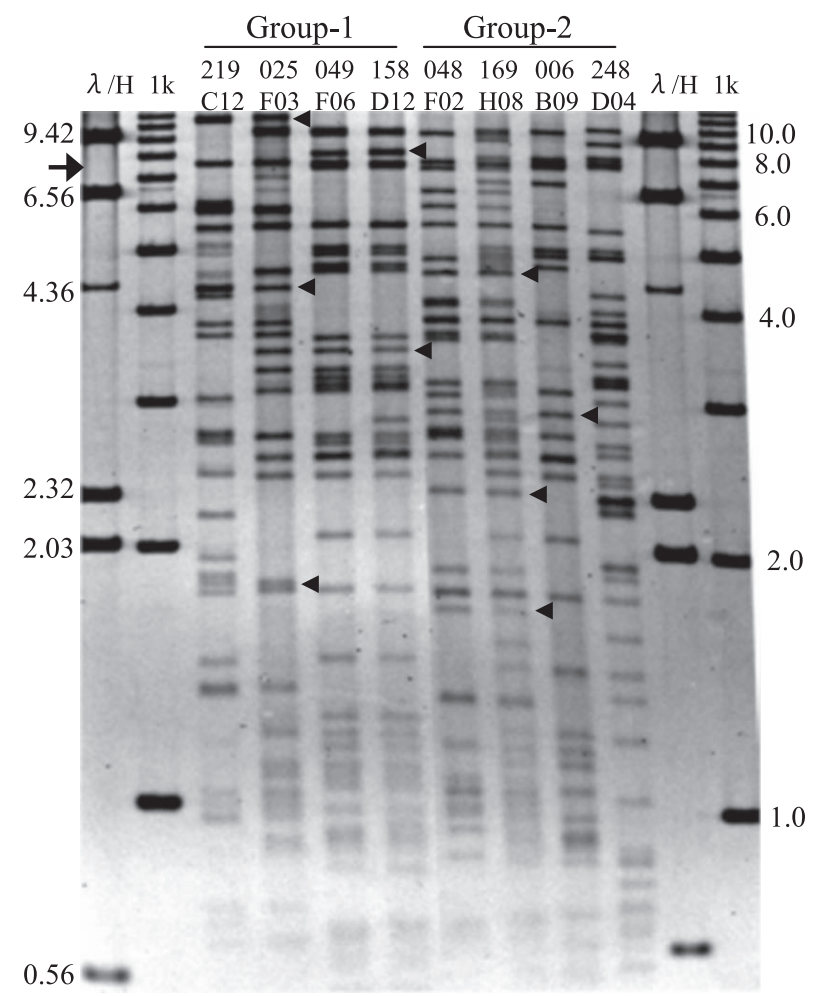

Fig. 4. BAC contig structure for the marker Mf0086 region. (A) BAC contig structure based on PCR amplification results and Hind III fingerprinting pattern. Horizontal lines denote each BAC clone, vertical dotted lines denote the relative position of BES and Mf0086 markers determined by PCR amplification. Each small box on horizontal lines represents the results of PCR amplification. (B) Restriction fragment pattern of Hind III-digested BAC clones. $\lambda / \mathrm{H}$ : lambda/Hind III marker, $1 \mathrm{k}$ : $1 \mathrm{~kb}$ DNA ladder (Takara). Arrowheads indicate bands specific to either group. An arrow indicates the vector-specific band $(7.5 \mathrm{~kb})$.

generated with 006B09M, 048F02M, and 048F02T, 11 heterozygous SNPs were detected by direct sequencing. Based on the SNP type of these marker sites, 8 BAC clones flanking Mf0086 were separated into two groups (Table 2). To confirm that the BAC clones in each group could be aligned into contig, BAC clones in each group were assembled separately by fingerprinting analysis. The fragment patterns of these BAC clones after digestion with Hind III are shown in Fig. 4B. Most fragments were present in both groups but some fragments were specifically detected on either group (those highlighted with an arrowhead); therefore, both groups were separately assembled into contigs. Assembling showed that each contig had a similar physical distance, 211 $\mathrm{kb}$ and $252 \mathrm{~kb}$, respectively. The alignment of BAC clones based on the amplification pattern with BES-derived primer pairs and Hind III digestion pattern confirmed the overlapping structure of the two BAC contigs, as shown in Fig. 4A.

\section{Mapping BES and CAPS markers with SNP}

To confirm the map position of the BAC contig containing marker Mf0086 on H-1 and M-1, the linkage relationship for the polyembryony locus with BES-derived SNP markers was analyzed. Of the SNP markers shown in Table 2, two sites (006B09M_150 and 048F02T_223) were heterozygous for 'Miyagawa wase' and homozygous for 'Kiyomi', and one SNP site (048F02T_223) was heterozygous for 'Harumi' 
Table 2. Classification of BAC clones by SNP types at BES sites

\begin{tabular}{|c|c|c|c|c|c|c|c|c|c|c|}
\hline \multirow{2}{*}{ Primer } & \multirow{2}{*}{ Pos. } & \multirow{2}{*}{$\begin{array}{l}\text { Direct } \\
\text { Seq }\end{array}$} & \multicolumn{4}{|c|}{ group-1 } & \multicolumn{4}{|c|}{ group-2 } \\
\hline & & & $219 \mathrm{C} 12$ & $025 \mathrm{~F} 03$ & 049F06 & 158D12 & 048F02 & $169 \mathrm{H} 08$ & 006B09 & 248D04 \\
\hline \multirow{4}{*}{$\begin{array}{l}\text { 006B09M } \\
\text { (439 bp) }\end{array}$} & 150 & $\mathrm{C} / \mathrm{T}$ & & & $\mathrm{T}$ & $\mathrm{T}$ & & $\mathrm{C}$ & $\mathrm{C}$ & $\mathrm{C}$ \\
\hline & 179 & $\mathrm{~A} / \mathrm{G}$ & & & G & G & & A & A & A \\
\hline & 267 & $\mathrm{C} / \mathrm{T}$ & & & $\mathrm{C}$ & $\mathrm{C}$ & & $\mathrm{T}$ & $\mathrm{T}$ & $\mathrm{T}$ \\
\hline & 290 & $\mathrm{C} / \mathrm{T}$ & & & $\mathrm{C}$ & $\mathrm{C}$ & & $\mathrm{T}$ & $\mathrm{T}$ & $\mathrm{T}$ \\
\hline \multirow{2}{*}{$\begin{array}{l}\text { 048F02M } \\
\text { (394 bp) } \\
\end{array}$} & 110 & $\mathrm{~A} / \mathrm{T}$ & & A & $\mathrm{A}$ & A & & $\mathrm{T}$ & $\mathrm{T}$ & $\mathrm{T}$ \\
\hline & 111 & $\mathrm{~A} / \mathrm{T}$ & & A & A & A & & $\mathrm{T}$ & $\mathrm{T}$ & $\mathrm{T}$ \\
\hline \multirow{5}{*}{$\begin{array}{l}\text { 048F02T } \\
(470 \mathrm{bp})\end{array}$} & 94 & $\mathrm{G} / \mathrm{T}$ & $\mathrm{T}$ & & & & G & G & & \\
\hline & 118 & $\mathrm{~A} / \mathrm{G}$ & A & & & & G & G & & \\
\hline & 223 & $\mathrm{C} / \mathrm{T}$ & $\mathrm{C}$ & & & & $\mathrm{T}$ & $\mathrm{T}$ & & \\
\hline & 272 & $\mathrm{C} / \mathrm{G}$ & $\mathrm{C}$ & & & & G & G & & \\
\hline & 409 & $\mathrm{~A} / \mathrm{G}$ & G & & & & A & A & & \\
\hline
\end{tabular}

and homozygous for F180. These SNP markers were segregated in each population (Table 3 ). In segregating populations $\mathrm{KM}$ and $\mathrm{FH}$, both of these SNP markers were mapped on linkage groups $\mathrm{M}-1$ and $\mathrm{H}-1$ (Fig. 4). In the linkage group $\mathrm{M}-1,006 \mathrm{~B} 09 \mathrm{M}$ 150 and 048F02T_223 were mapped on the nearest position $2.5 \mathrm{cM}$ distal to the polyembryony locus, and in the linkage group H-1, 048F02T_223 was completely linked with the polyembryony locus.

In the KM population, the monoembryony haplotype derived from 'Miyagawa wase' is shared between 'Kiyomi' and 'Miyagawa wase', indicating that the haplotype containing the allele linked in the coupling phase to the polyembryony locus corresponds to the polyembryony haplotype. On the two contigs containing marker Mf0086, contig-1, consisting of 219C12, 025F03, 049F06 and 158D12 (Fig. 4B), contained an allele linked in the coupling phase to the polyembryony locus on markers 006B09M_150 and 048F02T 223; therefore, contig- 1 can be considered to represent a polyembryony haplotype.

In addition to BES-derived markers, markers Lp0211 and Ks9001, which were mapped on both sides of the polyembryony locus in linkage group $\mathrm{H}-1$, were mapped in distal positions $2.9 \mathrm{cM}$ and $12.8 \mathrm{cM}$, respectively, from the polyembryony locus in linkage group M-1. The finding that the polyembryony locus was detected on the same marker interval is indicative of the commonality of the polyembryony locus in 'Miyagawa wase' and 'Harumi'.

The polyembryony locus was detected with the same marker alignment on $\mathrm{H}-1$ and $\mathrm{M}-1$, but the genetic distance on the flanking region of the polyembryony locus differed remarkably between $\mathrm{H}-1$ and $\mathrm{M}-1$. The genetic distances of the marker interval for Lp0211-048F02T, 048F02T-Ks9001, and Ks9001-Fb0103 were, respectively, 2.1, 4.3 and $2.1 \mathrm{cM}$ for $\mathrm{H}-1$ but $2.9,13.2$, and $7.0 \mathrm{cM}$ for M-1. The reason why the genetic distances differed between linkage groups is unclear, but the genetic distance of M-1 is consistently larger than H-1 and may result from the different genetic backgrounds of 'Miyagawa wase' and 'Harumi'.

\section{Discussion}

Previous studies have reported that one or a few genes are involved in the determination of polyembryony in the genus Citrus (Parlvliet and Cameron 1959, Iwamasa et al. 1967, Hong et al. 2001). In the present study, polyembryony was segregated in a binomial pattern fitting a $1: 1$ ratio in the $\mathrm{FH}$ population, suggesting segregation of a major gene that determines either polyembryony or monoembryony in this population. By mapping the polyembryony locus on $\mathrm{H}-1$ with CAPS markers, the co-segregating marker Mf0086 was characterized. This locus probably has a major effect on phenotypic expression of polyembryony in 'Harumi'.

To obtain genomic information about the regions flanking the polyembryony locus, the 'Miyagawa wase'-derived BAC library was screened for marker Mf0086, which was mapped in the nearest position to the polyembryony locus in 'Harumi', and BES-derived SNP markers were mapped on linkage group M-1 and H-1. The approach of using SNP

Table 3. Segregation of SNP markers in the KM and FH populations

\begin{tabular}{|c|c|c|c|c|c|c|c|c|c|c|}
\hline \multirow{2}{*}{ Marker } & \multicolumn{5}{|c|}{ KM populaton } & \multicolumn{5}{|c|}{ FH population } \\
\hline & $\mathrm{K}$ & M & heterozygous & homozygous & $\chi^{2}$ & $\mathrm{~F}$ & $\mathrm{H}$ & heterozygous & homozygous & $\chi^{2}$ \\
\hline Lp0211_148 & $\mathrm{A} / \mathrm{A}$ & $\mathrm{A} / \mathrm{G}$ & 28 & 12 & $6.40 * *$ & & & & & \\
\hline 006B09M_150 & $\mathrm{C} / \mathrm{C}$ & $\mathrm{C} / \mathrm{T}$ & 30 & 11 & $8.80^{* * *}$ & & & & & \\
\hline 048F02T_223 & $\mathrm{T} / \mathrm{T}$ & $\mathrm{C} / \mathrm{T}$ & 30 & 11 & $8.80^{* * *}$ & $\mathrm{~T} / \mathrm{T}$ & $\mathrm{C} / \mathrm{T}$ & 23 & 24 & $0.02 \mathrm{~ns}$ \\
\hline Ks9001_7̄77 & $\mathrm{A} / \mathrm{A}$ & $\mathrm{A} / \mathrm{G}$ & 16 & 25 & $1.97 \mathrm{~ns}$ & & & & & \\
\hline
\end{tabular}

ns: Not significant, $* *, * * *$ Significant at $1 \%$ and $0.1 \%$ level, respectively. 
markers to compare genetic linkage maps also indicated high SNP frequency among Citrus cultivars. The usage of SNPs on the BES and CAPS marker sites will be effective for obtaining linked markers for other important traits with a comparative genetic map approach in Citrus.

In linkage groups $\mathrm{M}-1$ and $\mathrm{H}-1$, the polyembryony locus was commonly detected on the nearest position to marker 048F02T_223 in the marker interval between Lp0211 and Ks9001. There is no direct evidence that polyembryony alleles in 'Miyagawa wase' of C. unshiu and in 'Harumi' from $C$. reticulata are common, but a highly conserved marker lineage suggests that the genomic structure of the polyembryony locus region is conserved in C. unshiu and C. reticulata, which may be indicative of conservation of the genomic structure of the polyembryony locus in a wide range of Citrus cultivars.

The marker lineage of the polyembryony locus was common in $\mathrm{M}-1$ and $\mathrm{H}-1$, but the genetic distance of the regions flanking the polyembryony locus differed remarkably, and the genetic distance of marker intervals on M-1 was consistently larger than on $\mathrm{H}-1$. This might be related to the different genetic background between 'Miyagawa wase' and 'Harumi'. In maize, a two-fold difference in genetic distance across $a 1$-sh 2 was observed among hybrid populations of different genetic background, and the relationship of transacting modifiers on meiotic recombination is indicated (Yandeau-Nelson et al. 2006). In Arabidopsis, the difference in meiotic recombination frequency among diverse accessions was indicated by examination of the chiasma frequency (Sanchez-Moran et al. 2002). Similar to other plant species, a genetic factor altering the recombination frequency may exist in Citrus and act differentially to change the recombination frequency between $\mathrm{M}-1$ and $\mathrm{H}-1$; however, to test this hypothesis further, experimentation with large segregating populations is required.

Several genomic studies on gametophytic apomixis in some grass species have been published (Akiyama et al. 2004, Ebina et al. 2005, Goel et al. 2006, Gualtieri et al. 2006). In these studies, the construction of high-density genetic linkage maps flanking the apomixis locus and isolation of BAC clones containing markers linked to the apomixis locus were performed, but positional cloning of the gene responsible has not been achieved due to the occurrence of recombination suppression in the flanking region (OziasAkins and van Dijk 2007). In contrast, several recombinations were detected in the flanking region of the polyembryrony locus in linkage groups $\mathrm{M}-1$ and $\mathrm{H}-1$. The occurrence of recombination in the flanking region of the polyembryony locus suggests the possibility of identifying the responsive gene by positional cloning. It is also suggested that the haplotype-specific BAC contig containing marker Mf0086 will help to identify the genes responsible for polyembryony and to obtain genomic information on the regions flanking the polyembryony locus. Comparative study of the genomic sequence of BAC clones located at the polyembryony locus and previously characterized sequences near the apomixis locus in grass species (Calderini et al. 2006) would provide information about the extent of similarity of the genomic sequences between the different types of apomixis, thus resulting in a better understanding of apomictic reproduction.

\section{Acknowledgements}

This study was supported by Research Fellowships from the Japan Society for the Promotion of Science for Young Scientists.

\section{Literature Cited}

Akiyama,Y., J.A.Conner, S.Goel, D.T.Morishige, J.E.Mullet, W.W. Hanna and P.Ozias-Akins (2004) High-resolution physical mapping in Pennisetum squamulatum reveals extensive chromosomal heteromorphism of the genomic region associated with apomixis. Plant Physiol. 134: 1733-1741.

Bernet,G.P., M.P.Bretó and M.J.Asins (2004) Expressed sequence enrichment for candidate gene analysis of citrus tristeza virus resistance. Theor. Appl. Genet. 108: 592-602.

Calderini, O., S.B.Chang, H.de Jong, A.Busti, F.Paolocci, S.Arcioni, S.C. de Vries, M.H.C. Abma-Henkens, R.M.K. Lankhorst, I.S.Donnison and F.Pupilli (2006) Molecular cytogenetics and DNA sequence analysis of an apomixis-linked BAC in Paspalum simplex reveal a non pericentromere location and partial microcolinearity with rice. Theor. Appl. Genet. 112: 1179-1191.

Carlos de Oliveira,A., M.Bastianel, M.Cristofani-Yaly, A. Morais do Amaral and M.A.Machado (2007) Development of genetic maps of the citrus varieties 'Murcott' tangor and 'Pera' sweet orange by using fluorescent AFLP markers. J. Appl. Genet. 48: 219-231.

Carman,J.G. (1997) Asynchronous expression of duplicate genes in angiosperms may cause apomixis, bispory, tetraspory, and polyembryony. Biol. J. Linn. Soc. 61: 51-94.

Chen,C., K.Bowman, Y.Choi, P.Dang, M.Rao, S.Huang, J.Soneji, T.McCollum and F.Gmitter (2008) EST-SSR genetic maps for Citrus sinensis and Poncirus trifoliata. Tree Genetics \& Genomes 4: $1-10$.

Deng,Z., S.Huang, P.Ling, C.Yu, Q.Tao, C.Chen, M.K.Wendell, H.B.Zhang and F.G.Gmitter (2001) Fine genetic mapping and BAC contig development for the citrus tristeza virus resistance gene locus in Poncirus trifoliata (Raf.). Mol. Genet. Genomics 265: 739-747.

Durham,R.E., P.C.Liou, F.G.Gmitter and G.A.Moore (1992) Linkage of restriction fragment length polymorphisms and isozymes in Citrus. Theor. Appl. Genet. 84: 39-48.

Ebina, M., H.Nakagawa, T. Yamamoto, H.Araya, S. Tsuruta, M. Takahara and K.Nakajima (2005) Co-segregation of AFLP and RAPD markers to apospory in Guineagrass (Panicum maximum Jacq.). Grassl. Sci. 51: 71-78.

Gmitter,F., C.Chen, M.Nageswara Rao and J.Soneji (2007) Citrus fruits. In: Kole,C. (ed.) Genome Mapping and Molecular Breeding in Plants, Volume 4 Fruits and Nuts, Springer-Verlag, Berlin, pp. 265-279.

Goel,S., Z.Chen, Y.Akiyama, J.A.Conner, M.Basu, G. Gualtieri, W.W.Hanna and P.Ozias-Akins (2006) Comparative physical mapping of the apospory-specific genomic region in two apomictic grasses: Pennisetum squamulatum and Cenchrus ciliaris. Genetics 173: $389-400$.

Gualtieri,G., J.A.Conner, D.T.Morishige, L.D.Moore, J.E.Mullet and 
P.Ozias-Akins (2006) A segment of the apospory-specific genomic region is highly microsyntenic not only between the apomicts Pennisetum squamulatum and buffelgrass, but also with a rice chromosome 11 centromeric-proximal genomic region. Plant Physiol. 140: 963-971.

Hong,Q.B., S.Q.Xiang, K.L.Chen and L.G.Chen (2001) Two complementary dominant genes controlling apomixis in genus Citrus and poncirus. Yi Chuan Xue Bao 28: 1062-1067.

Jarrell,D.C., M.L.Roose, S.N.Traugh and R.S.Kupper (1992) A genetic map of citrus based on the segregation of isozymes and RFLPs in an intergeneric cross. Theor. Appl. Genet. 84: 49-56.

Iwamasa,M., I.Ueno and M.Nishiura (1967) Inheritance of nucellar embryony in Citrus. Bull. Hort. Res. Sta. Japan, ser. B. 7: 1-10.

Kobayashi,S., I.Ikeda and M.Nakantani (1978) Studies on the nucellar embryogenesis in Citrus I. Formation of nucellar embryo and development of ovule. Bull. Fruit Tree Res. Stn. E. 2: 9-24.

Kobayashi,S., I.Ikeda and M.Nakatani (1979) Studies on nucellar embryogenesis in Citrus. II. Formation of the primordium cell of the nucellar embryo in the ovule of the flower bud, and its meristematic activity. J. Jpn. Soc. Hort. Sci. 48: 179-185.

Koltunow,A.M. (1993) Apomixis: Embryo sacs and embryos formed without meiosis or fertilization in ovules. Plant Cell 5: 1425-1437.

Nakano,M., T.Shimizu, T.Kuniga, H.Nesumi and M.Omura (2008) Mapping and haplotyping of the flanking region of the polyembryony locus in Citrus unshiu Marcow. J. Jpn. Soc. Hort. Sci. 77: 109114.

Omura,M., T.Ueda, M.Kita, A.Komatsu, Y.Takanokura, T.Shimada, T.Endo-Inagaki, H.Nesumi and T.Yoshida (2003) EST mapping in Citrus. International Citrus Congress (9th: 2000: Orlando, Florida), 2003: 71-74.

Ozias-Akins,P. and P.J.van Dijk (2007) Mendelian genetics of apomixis in plants. Annu. Rev. Genet. 41: 509-537.

Parlevliet,J.E. and J.W.Cameron (1959) Evidence on the inheritance of nucellar embryony in Citrus. Proc. Am. Soc. Hortic. Sci. 74: 252-260.

Patocchi,A., B.A.Vinatzer, L.Gianfranceschi, S.Tartarini, H.B.Zhang, S.Sansavini and C.Gessler (1999) Construction of a $550 \mathrm{~kb}$ BAC contig spanning the genomic region containing the apple scab re- sistance gene $V f$. Mol. Genet. Genomics 262: 884-891.

Sanchez-Moran,E., S.J.Armstrong, J.L.Santos, F.C.H.Franklin and G.H.Jones (2002) Variation in chiasma frequency among eight accessions of Arabidopsis thaliana. Genetics 162: 1415-1422.

Shimada,T., T.Yamamoto, H.Hayama, M.Yamaguchi and T.Hayashi (2000) A genetic linkage map constructed by using an intraspecific cross between peach cultivars grown in Japan. J. Jpn. Soc. Hort. Sci. 69: 536-542.

Shimada,T., F.Nishikawa, T.Endo, H.Fujii, Y.Nobata, T.Shimizu and M.Omura (2005) Construction and initial evaluation of BAC library for Citrus unshiu Marc. 'Miyagawa wase'. J. Jpn. Soc. Hort. Sci. 74 (Suppl.1): 187.

Shizuya,H., B.Birren, U.J.Kim, V.Mancino, T.Slepak, Y.Tachiiri and M.Simon (1992) Cloning and stable maintenance of 300-kilobasepair fragments of human DNA in Escherichia coli using an Ffactor-based vector. Proc. Natl. Acad. Sci. USA 89: 8794-8797.

Telgmann-Rauber,A., A.Jamsari, M.S.Kinney, J.C.Pires and C.Jung (2007) Genetic and physical maps around the sex-determining $M$ locus of the dioecious plant asparagus. Mol. Genet. Genomics 278: 221-234.

Ueno,I., M.Iwamasa and M.Nishiura (1967) Embryo number of various varieties of Citrus and its relatives. Bull. Hort. Res. Sta. Japan, ser. B. 7: 11-22.

Yandeau-Nelson,M.D., B.J.Nikolau and P.S.Schnable (2006) Effects of trans-acting genetic modifiers on meiotic recombination across the al-sh2 interval of maize. Genetics 174: 101-112.

Yang, Z.N., X.R.Ye, S.Choi, J. Molina, F. Moonan, R.A. Wing, M.L.Roose and T.E.Mirkov (2001) Construction of a 1.2-Mb contig including the citrus tristeza virus resistance gene locus using a bacterial artificial chromosome library of Poncirus trifoliata (L.) Raf. Genome 44: 382-393.

Yang,Z.N., X.R.Ye, J.Molina, M.L.Roose and T.E.Mirkov (2003) Sequence analysis of a 282-kilobase region surrounding the citrus tristeza virus resistance gene (Ctv) locus in Poncirus trifoliata L. Raf. Plant Physiol. 131: 482-492.

Yin,J., W.Guo, L.Yang, L.Liu and T.Zhang (2006) Physical mapping of the $R f 1$ fertility-restoring gene to a $100 \mathrm{~kb}$ region in cotton. Theor. Appl. Genet. 112: 1318-1325. 